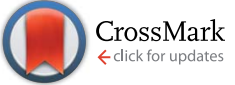

Cite this: RSC Adv., 2017, 7, 3660

Received 6th October 2016

Accepted 17th November 2016

DOI: $10.1039 / \mathrm{c} 6 \mathrm{ra} 24816 \mathrm{k}$

www.rsc.org/advances

\title{
Trypsin and chymotrypsin are necessary for in vitro enzymatic digestion of rice starch
}

\begin{abstract}
Shujun Wang, ${ }^{\text {aab }}$ Peiyan Li, ${ }^{a}$ Teng Zhang, ${ }^{a}$ Shuo Wang ${ }^{\star a b}$ and Les Copeland ${ }^{\mathrm{c}}{ }^{\mathrm{C}}$
An appropriate in vitro starch digestion model is necessary to comparatively evaluate the digestibility of starch samples and to help predict the in vivo glycemic responses of starchy foods. In the present study, the effect of trypsin and chymotrypsin on in vitro digestion of rice starch was studies for the first time. Inclusion of the trypsin and chymotrypsin in the digestion protocols, to more closely simulate intestinal digestion, increased the hydrolysis percentage of cooked rice and rice starch gels. The hydrolysis kinetics results showed that the digestion rate of cooked rice and starch gels was increased with the addition of trypsin and chymotrypsin in the simulated intestinal fluids. This study showed clearly that the addition of proteases during simulated intestinal digestion is necessary to evaluate accurately the in vitro digestibility of starch or starchy foods.
\end{abstract}

\section{Introduction}

The digestibility of starch is of considerable nutritional interest in relation to the increasing incidence of obesity and dietrelated diseases. A marked increase in postprandial glucose in the bloodstream following consumption of a starch-rich meal results mainly from the amylolytic breakdown of starch in the digestive tract to glucose. ${ }^{1}$ Rice is one of the most important cereal grains and a staple food of over half the world's population. As the primary dietary source of carbohydrate for many people, rice plays an important role in meeting energy requirements and nutrient intake. Rice mostly contains starch, and to a lesser extent, protein, lipids and numerous minor constituents. Starch contributes $50-70 \%$ of the dietary energy and is the most abundant glycemic carbohydrate in the human diet. ${ }^{2}$ As cooked rice starch is normally hydrolyzed readily by amylases, it is usually considered as a high Glycemic Index (GI) food. ${ }^{3}$ With the number of diabetes patients predicted to increase from 171 million in 2000 to 366 million in 2030, ${ }^{4}$ a better understanding of the digestibility of starch in cooked rice is particularly important for those eating rice as a staple food.

\footnotetext{
${ }^{a}$ Key Laboratory of Food Nutrition and Safety, Ministry of Education, College of Food Engineering and Biotechnology, Tianjin University of Science \& Technology, No. 29, $13^{\text {th }}$ Avenue, Tianjin Economic and Developmental Area (TEDA), Tianjin 300457, China.E-mail:sjwang@tust.edu.cn; s.wang@tust.edu.cn; Tel: +86-22-60912486

${ }^{b}$ Beijing Advanced Innovation Center for Food Nutrition and Human Health, Beijing Technology \& Business University, Beijing 100048, China

${ }^{c}$ Faculty of Agriculture and Environment, The University of Sydney, NSW 2006, Australia

$\dagger$ School of Life and Environmental Sciences, University of Sydney, NSW, Australia 2006.
}

Starch digestibility can be assessed by in vitro or in vivo methods, which are complementary rather than alternatives. In vitro digestion models measure the physico-chemical processes of starch breakdown, whereas in vivo methods, such as measuring the GI in animals or humans, reflect a physiological process that includes starch breakdown, glucose absorption into and clearance from the bloodstream. ${ }^{5}$ In vivo methods more accurately reflect the physiology of starch digestion, but they are complicated, time-consuming and expensive. ${ }^{6}$ Hence, much effort has been devoted to the development of various in vitro procedures for starch digestion. ${ }^{6}$ In practice, in vitro models provide a useful alternative to in vivo methods for rapidly screening the digestibility of food ingredients. However, the in vitro models do not reflect accurately the inherent complexity of in vivo digestive processes, ${ }^{1,7-9}$ they are nevertheless very useful for comparing the relative digestibility of native and cooked starches, for example in plant breeding or food product development.

Starch digestion in the human digestive tract typically involves oral, gastric and intestinal phases. Residual starch not digested in these phases is subject to fermentation in the large intestine..$^{\mathbf{8 1 1 - 1 3}}$ In adults, most of the starch molecules are broken down in the duodenum, after passing through the acidic environment of the stomach. ${ }^{14}$ In infants, more of the starch digestion is accomplished by salivary amylases. ${ }^{15}$ The most widely adopted approach for measuring starch digestion in vitro is based on the protocol developed by Englyst (1992), ${ }^{\mathbf{1 6}}$ who classified starch into three fractions: rapidly digested, slowly digested and resistant starches, according to the amount of glucose released at certain time intervals by controlled enzymic hydrolysis of starchy foods with pancreatin and amyloglucosidase. There have been many subsequent modifications to the original method, including substantial 
variations in ratio of enzyme to substrate, using different combinations or amounts of enzyme activities, composition and $\mathrm{pH}$ of reaction medium, stirring rate, and incubation temperature and incubation time..$^{\mathbf{6 , 8 , 1 3 , 1 7 , 1 8}}$ A simulated gastric digestion phase using pepsin under acidic conditions is sometimes included, especially for digestion of starchy plant foods. ${ }^{4,7,19,20}$ It is also pertinent to note that pancreatin, as the exocrine secretion pancreatic cells, contains a mixture of enzyme activities including proteases, amylases, lipases and ribonucleases. The amounts of these activities in commercial pancreatin preparations vary according to source and grade of the preparation. Hence, the inclusion of pancreatin, along with added amylases and amyloglucosidases in starch breakdown studies, introduces a further source of the variation between experimental protocols that causes difficulties in the comparability between different studies.

In a recent review, a standardized in vitro starch digestion method was proposed, with trypsin and chymotrypsin, the main proteolytic enzymes in the small intestine, recommended to be included in simulated intestinal digestion. ${ }^{13}$ However, little specific attention has been given to the role the main proteolytic enzymes in the small intestine, namely trypsin and chymotrypsin, may play in starch digestion. In the present study, we aim to evaluate the effect of including proteases, especially during simulated intestinal digestion, on enzyme digestibility of starch. Cooked rice and rice starch gel were used as the test materials, as these are the primary dietary of carbohydrate for a large proportion of the world's population.

\section{Materials and methods}

\section{Materials}

Milled indica hybrid rice (Hua liangyou) was kindly provided by Huazhong Agricultural University (Wuhan, China). Starch was isolated from rice according to a method described by Spigno and Faveri, (2004). ${ }^{21}$ Porcine pancreatic $\alpha$-amylase (PPA, A3176, EC 3.2.1.1, type VI-B from porcine pancreas, 15 units per $\mathrm{mg}$ ), pepsin (P7012, pepsin from porcine gastric mucosa, $\geq 2500$ units per $\mathrm{mg}$ ), trypsin (T0303, trypsin from porcine pancreas Type IX-S, lyophilized powder, 13 000-20 000 BAEE units per $\mathrm{mg}$ protein) and $\alpha$-chymotrypsin (C4129, $\alpha$ chymotrypsin from bovine pancreas Type II, lyophilized power, $\geq 40$ units per mg protein) were purchased from Sigma Chemical Co. (St. Louis, Mo., USA). Amyloglucosidase (AMG, $3260 \mathrm{U} \mathrm{mL}^{-1}$ ), Total Starch Assay Kit and D-Glucose Assay Kit (glucose oxidase/peroxide, GOPOD format) were purchased from Megazyme International Ireland Ltd. (Bray County, Wicklow, Ireland). Other chemical reagents were all of analytical grade.

\section{Composition of rice grains and isolated starches}

Rice grains were ground using a household high-speed multi-function grinder (BJ-350, Deqing Baijie Electrical Appliance Co. Ltd, China) and passed through a $0.5 \mathrm{~mm}$ sieve. The resulting rice flour was used for compositional analysis. Total starch content of rice grains and rice starch were determined using the Total Starch Assay Kit following the procedure provided by the manufacture. The nitrogen content of rice grains and starch were determined by standard Kjeldahl methodology. Protein content was estimated by multiplying the nitrogen content by a conversion factor of 6.25. Apparent amylose content and lipid content of rice grains and starch were determined according to Chrastil $(1987)^{22}$ and the AACC-International Approved Method 44-15.02, ${ }^{23}$ respectively. All analyses were performed in triplicate.

\section{Preparation of cooked rice and starch gels}

Cooked rice was prepared by steaming rice grains following the combined methods of Lu et al. (2013) and Kang et al. (2003) with some modifications. ${ }^{24,25}$ Rice grains $(10 \mathrm{~g})$ were weighed into an aluminum canister, and distilled water was added to give a rice : water ratio of $1: 1(\mathrm{w} / \mathrm{v})$. The canister was covered with a sheet of aluminum foil and placed immediately on a holder in a household rice cooker (WZA-0512, Shunde Electrical Appliance Co. Ltd. Guangdong, China), containing $400 \mathrm{~mL}$ water. The rice was cooked for $10 \mathrm{~min}$, followed by a $10 \mathrm{~min}$-holding period at the warming setting of the cooker. The cooked rice grains were allowed to cool at room temperature for $20 \mathrm{~min}$ before in vitro digestion tests.

The starch gel was prepared by weighing starch $(100 \mathrm{mg}$, dry weight basis) into a $50 \mathrm{~mL}$ centrifuge tube and distilled water was added to obtain a starch : water ratio of $1: 1$. The starchwater mixture was placed into the rice cooker and cooked according to the conditions described for cooking rice. After cooking, the starch gel was allowed to cool at room temperature for $20 \mathrm{~min}$ before in vitro digestion tests.

\section{In vitro starch digestibility}

Four different protocols were designed to evaluate the role of proteases in in vitro digestibility of cooked rice and rice starch gels. As represented schematically in Fig. 1, Protocol 1, which is based on Englyst's method, ${ }^{16}$ involved porcine pancreatic $\alpha$ amylase (PPA) and amyloglucosidase (AMG) as the hydrolytic enzymes, whereas the enzyme mix for Protocol 2 included trypsin and chymotrypsin in addition to PPA and AMG. Protocols 1 and 2 are referred to subsequently as simulating intestinal digestion. Protocols 3 and 4 had the same enzyme mixtures as Protocols 1 and 2, respectively, except that an acidic pepsin step was included prior to the main digestion phase. Protocols 3 and 4 are referred to subsequently as simulating gastro-intestinal digestion. The acidic pepsin phase of Protocol 3 and 4 is referred to as simulating gastric digestion.

PPA solution was prepared by suspending $1.3 \mathrm{~g}$ of PPA in 12 $\mathrm{mL}$ of water at $37{ }^{\circ} \mathrm{C}$ with magnetic stirring for $10 \mathrm{~min}$. The mixture was centrifuged at $2000 \mathrm{~g}$ for $10 \mathrm{~min}$ and $0.1 \mathrm{~mL}$ of amyloglucosidase was added to $8.0 \mathrm{~mL}$ of the supernatant. Protease solutions were prepared by dissolving pepsin (1 $\mathrm{mg}$ $\mathrm{mL}^{-1}$ ) in $\mathrm{HCl}$ solution (0.01 M, pH 2), trypsin (2995 $\left.\mathrm{U} \mathrm{mL}^{-1}\right)$ and $\alpha$-chymotrypsin $\left(18 \mathrm{U} \mathrm{mL}^{-1}\right)$ in acetate buffer $(0.2 \mathrm{M}, \mathrm{pH}$ ), respectively. All enzyme solutions were prepared freshly before utilization. 

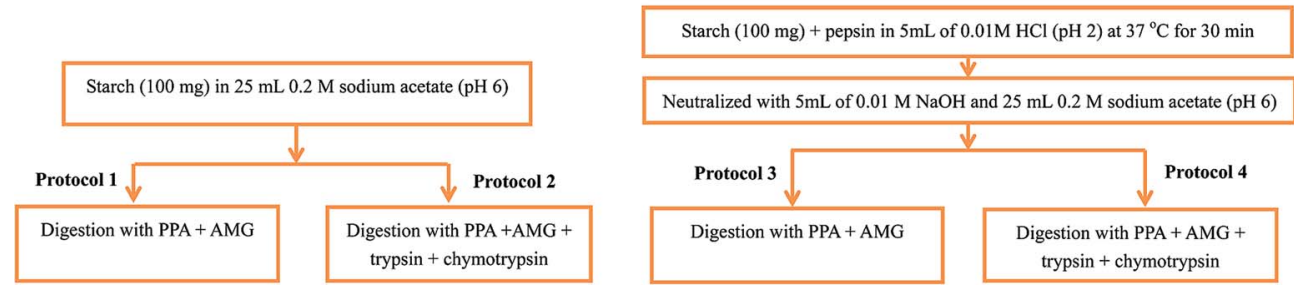

Fig. 1 Schematic representation of the four in vitro starch digestion protocols. The details are described in the Methods.

\section{Simulated intestinal digestion protocols}

The cooked rice grains or starch gels (both containing $100 \mathrm{mg}$ starch, dry weight basis) were transferred to a $50 \mathrm{~mL}$ centrifuge tube and mixed with $25 \mathrm{~mL}$ sodium acetate buffer $(0.2 \mathrm{M}, \mathrm{pH}$ 6). For Protocol 1, an enzyme mixture containing 8228 U PPA and $204 \mathrm{U}$ AMG in $5 \mathrm{~mL}$ was added. For Protocol 2, $1 \mathrm{~mL}$ of trypsin $(2995 \mathrm{U})$ and $1 \mathrm{~mL}$ of $\alpha$-chymotrypsin (18 U) solutions were added to the enzyme mixture used in Protocol 1 . The rice and starch gels were incubated with stirring at $260 \mathrm{rpm}$ at $37^{\circ} \mathrm{C}$ and aliquots $(0.2 \mathrm{~mL})$ were taken at 20, 40, 60, 80, 100 and $120 \mathrm{~min}$. Enzyme reactions were stopped by mixing the aliquots with 0.8 $\mathrm{mL}$ of absolute ethanol and centrifuging at $14500 \mathrm{~g}$ for $3 \mathrm{~min}$. The supernatant was used to determine the glucose content by the Megazyme GOPOD kit.

\section{Simulated gastro-intestinal digestion protocols}

The cooked rice grains or starch gels (both containing $100 \mathrm{mg}$ starch, dry weight basis) were transferred to a $50 \mathrm{~mL}$ centrifuge tube, and incubated in $5 \mathrm{~mL}$ of a solution containing pepsin (12 $500 \mathrm{U})$ in $0.01 \mathrm{M} \mathrm{HCl}(\mathrm{pH} 2)$ at $37^{\circ} \mathrm{C}$ with magnetic stirring (260 rpm) for $30 \mathrm{~min}$. Pepsin action was stopped by neutralizing the solution with $5 \mathrm{~mL} 0.01 \mathrm{M} \mathrm{NaOH}$ and $25 \mathrm{~mL}$ of $0.2 \mathrm{M}$ sodium acetate buffer ( $\mathrm{pH}$ 6.0) was added. For Protocol 3, $5 \mathrm{~mL}$ of a mixture containing PPA (8228 U) and AMG (204 U) was added to the above mixture. For Protocol 4, $1 \mathrm{~mL}$ trypsin $(2995$ $\mathrm{U})$ and $1 \mathrm{~mL} \alpha$-chymotrypsin $(18 \mathrm{U})$ enzyme solutions were further added to the above mixture used in Protocol 3. The mixtures were incubated with stirring at $260 \mathrm{rpm}$ at $37^{\circ} \mathrm{C}$ for $120 \mathrm{~min}$. Aliquots $(0.2 \mathrm{~mL})$ were taken after $30 \mathrm{~min}$ (G30) of pepsin digestion, and from the simulated intestinal digestions at 20, 40, 60, 80, 100 and $120 \mathrm{~min}$. The aliquots were processed for glucose determination as described above. Results were represented as percentage of starch hydrolysis calculated from the amount of glucose produced (multiplied by 0.9 to convert to the anhydro-glucosyl form) divided by the initial amount of starch.

The amount of starch digested after $20 \mathrm{~min}$ (referred to as rapidly digested starch, RDS), between $20 \mathrm{~min}$ and $120 \mathrm{~min}$ (referred to as slowly digested starch, SDS), and starch remaining after $120 \mathrm{~min}$ (referred to as resistant starch, RS), according to the terminology developed by Englyst et al. (1992), ${ }^{16}$ were calculated.

\section{First-order kinetics}

As in vitro starch hydrolysis may be represented as a first order kinetic process, the digestograms of starch hydrolysis were fitted to the first-order rate equation. ${ }^{26}$

$$
C_{t}=C_{\infty}\left(1-\mathrm{e}^{-k t}\right)
$$

where $C_{t}$ is the amount of starch digested at time $t, C_{\infty}$ is the estimated amount of starch digested at the reaction end point, and $k$ is the first order rate constant. ${ }^{1}$ For ease of interpretation, $C_{t}$ may be expressed as the percentage of starch hydrolysed. The value of $k$ can be calculated from the slope of a linear-leastsquares fit of a plot of $\ln \left(1-C / C_{\infty}\right)$ against $t$.

Samples taken after $30 \mathrm{~min}$ of pepsin digestion and after $120 \mathrm{~min}$ of simulated intestinal digestion were used for morphological observations. The samples were centrifuged at $14500 \mathrm{~g}$ for $3 \mathrm{~min}$, washed twice with absolute ethanol followed by distilled water for three times and freeze-dried.

\section{Scanning electron microscope (SEM)}

The freeze-dried digestion samples were mounted on a stub with double-sided adhesive tapes, and coated with gold in a sputter coater (JEC-3000FC, Tokyo, Japan). The samples were imaged using a scanning electron microscope (JSM-IT300LV, JEOL, Japan) at an accelerating voltage of $10 \mathrm{kV}$.

\section{Statistical analysis}

Results are reported as the mean values and standard deviations of at least duplicate measurements. Analysis of variance (ANOVA) by Duncan's test $(p<0.05)$ were conducted using the SPSS 19.0 statistical Software Program (SPSS Inc. Chicago, IL, USA).

\section{Results and discussion}

\section{Basic composition of rice grains and isolated starches}

The total starch and protein, apparent amylose content and lipid contents of the rice grains and isolated starches are summarized in Table 1 . The values obtained are in general agreement with previous reports., ${ }^{4,27-31}$ 
Table 1 Chemical composition of rice grains and isolated starch $^{a}$

\begin{tabular}{lcc}
\hline Samples & Rice grains & Isolated starch \\
\hline Total starch & $72.8 \pm 1.6$ & $89.5 \pm 0.4$ \\
Crude protein & $8.9 \pm 0.1$ & $0.04 \pm 0.01$ \\
Apparent amylose & $8.1 \pm 0.2$ & $13.1 \pm 0.2$ \\
content & & \\
Lipid & $0.8 \pm 0.1$ & $0.03 \pm 0.00$
\end{tabular}

${ }^{a}$ All values are $\%$ dry basis and are means \pm standard deviations of triplicate determinations.

\section{In vitro enzymatic digestibility}

Digestograms and the fit of the data to first order kinetic equation, for starch hydrolysis in cooked rice and gels using the different in vitro digestion protocols are shown in Fig. 2. The digestograms of cooked rice were characterized by an initial rapid digestion rate followed by a more gradual rate of digestion, reaching a plateau after 60-80 min during the intestinal digestion process (Fig. 2A). The digestograms for starch gels using Protocols 1, 2 and 3 did not reach a plateau, whereas Protocol 4 gave a digestogram more similar to the cooked rice samples (Fig. 2B). Starch in cooked rice and starch gels was hydrolyzed to the greatest extent using Protocol 4 compared to
Protocols 1, 2 and 3. Differences between Protocols 1, 2 and 3 were not significant for cooked rice, whereas Protocol 3 was slightly more effective than Protocols 1 and 2 for the starch gel (Table 2). The RDS fraction increased from 53\% (Protocol 1) to $64 \%$ (Protocol 4) for cooked rice. Slowly digested starch (SDS) content decreased from $45 \%$ to $35 \%$ (Table 2). The RDS content of starch gels increased from $11 \%$ to $34 \%$ and enzyme-resistant starch (RS) decreased from $56 \%$ to $21 \%$ (Table 2 ). Over $95 \%$ of the starch in cooked rice was digested after $2 \mathrm{~h}$ of amylolysis in all four protocols (Fig. 2A). In comparison, the amount of starch hydrolyzed in the gels after two hours of amylolysis ranged from 44\% (Protocol 1) to 79\% (Protocol 4) (Fig. 2B).

\section{Effect of pepsin on the starch digestibility}

No glucose release from starch was detected from both cooked rice and starch gels during the acidic pepsin incubation in Protocols 3 and 4. However, the simulated gastric digestion increased the digestibility of starch in cooked rice in the subsequent simulated intestinal digestion stage. Hence, our results indicated that using a simulated intestinal digestion protocol alone may underestimate starch susceptibility to amylolysis.

Gelatinized starch in cooked rice is embedded in, or interacts with, a protein matrix to form an extended three-dimensional network. ${ }^{18,32}$ The protein matrix and interaction between starch
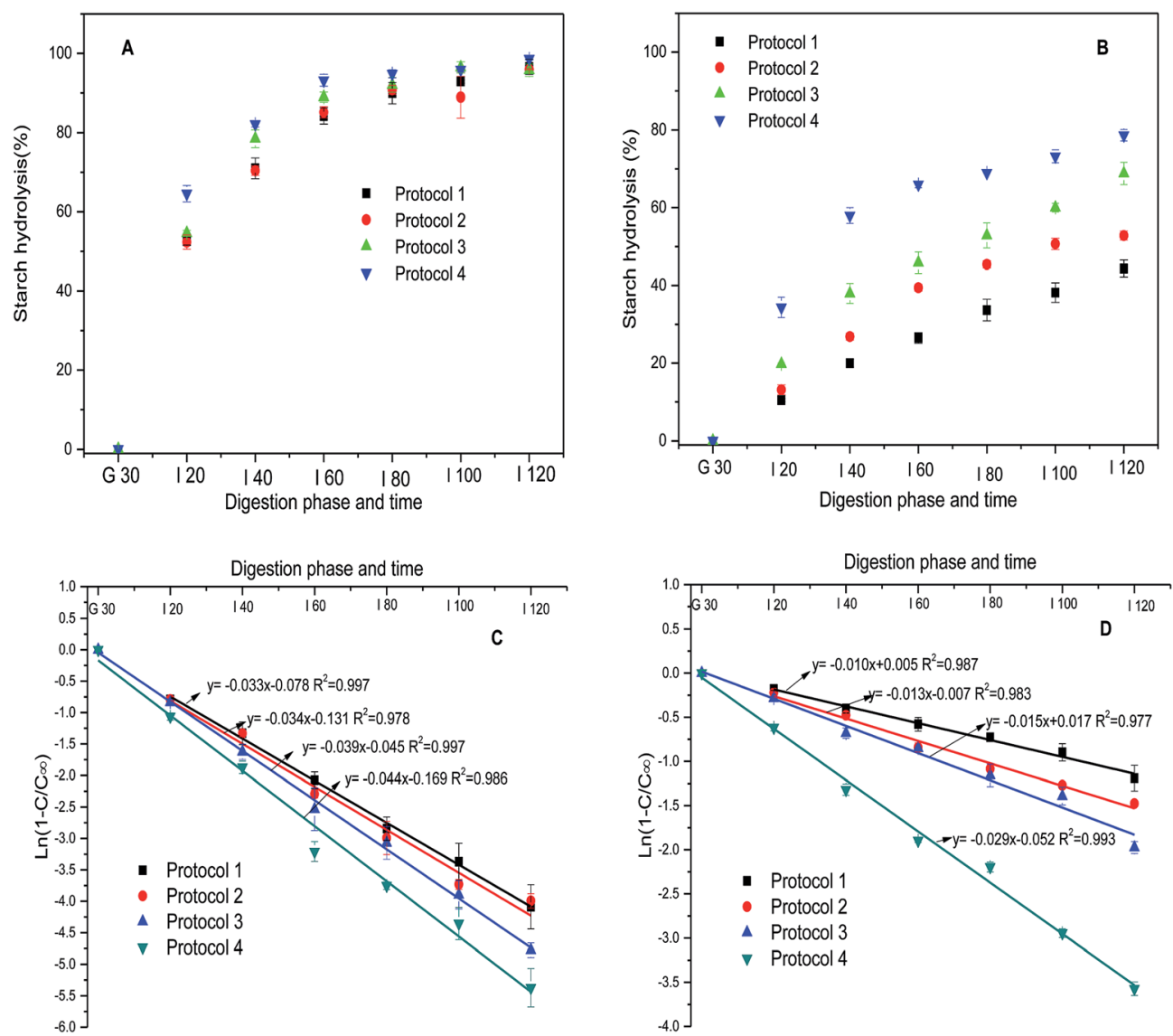

Fig. 2 Digestibility curves of cooked rice (A) and starch gels (B); fitting of first order kinetics to cooked rice (C) and starch gels (D). G: simulated gastric stage, I: simulated intestinal digestion stage. The number followed $\mathrm{G}$ or I on the $X$ axis present elapsed time in minutes for each stage. 
Table 2 Rapidly digestible starch (RDS), slowly digestible starch (SDS), resistant starch (RS) and kinetics parameter of starch hydrolysis percentage of the cooked rice and rice starch gels ${ }^{a}$

\begin{tabular}{|c|c|c|c|c|c|}
\hline Samples & RDS (\%) & SDS (\%) & RS (\%) & $C_{\infty}(\%)$ & $k\left(\min ^{-1}\right)$ \\
\hline \multicolumn{6}{|l|}{ CR } \\
\hline Protocol 1 & $52.6 \pm 1.0 \mathrm{a}$ & $44.8 \pm 2.8 b$ & $2.6 \pm 1.8 \mathrm{ab}$ & $96.3 \pm 1.5 \mathrm{a}$ & $0.033 \pm 0.001 \mathrm{a}$ \\
\hline Protocol 3 & $54.6 \pm 0.7 \mathrm{a}$ & $40.3 \pm 0.0 \mathrm{~b}$ & $5.1 \pm 0.7 b$ & $96.4 \pm 1.5 \mathrm{a}$ & $0.039 \pm 0.001 \mathrm{ab}$ \\
\hline Protocol 4 & $63.6 \pm 1.4 \mathrm{~b}$ & $35.1 \pm 1.2 \mathrm{a}$ & $1.4 \pm 0.2 \mathrm{a}$ & $97.2 \pm 0.9 \mathrm{a}$ & $0.044 \pm 0.002 \mathrm{~b}$ \\
\hline Protocol 2 & $13.1 \pm 1.3 \mathrm{a}$ & $39.7 \pm 0.2 b$ & $47.2 \pm 1.2 \mathrm{c}$ & $70.2 \pm 3.7 \mathrm{a}$ & $0.013 \pm 0.000 \mathrm{a}$ \\
\hline Protocol 3 & $19.8 \pm 0.3 b$ & $49.1 \pm 2.6 c$ & $31.2 \pm 2.9 b$ & $83.1 \pm 0.2 c$ & $0.015 \pm 0.001 \mathrm{a}$ \\
\hline Protocol 4 & $34.4 \pm 2.6 c$ & $44.3 \pm 3.5 b c$ & $21.3 \pm 1.5 \mathrm{a}$ & $78.3 \pm 1.6 b c$ & $0.034 \pm 0.001 b$ \\
\hline
\end{tabular}

${ }^{a}$ Mean \pm standard deviations from duplicate measurements are presented; values for each set of samples with different letters in the same column are significantly different $(p \leq 0.05)$. CR: cooked rice, without prior soaking, SG: starch gels, $C_{\infty}$ : the estimated amount of starch digested at the end of the reaction, $k$ : first order rate kinetic constant.

and protein can act as a barrier to block access of amylolytic enzymes to starch, hence reducing its digestibility. ${ }^{32-34}$ During simulated gastric digestion by pepsin, hydrolysis of the protein matrix and/or disruption of interactions between starch and protein may increase the accessibility of starch to amylolytic enzymes by releasing more swollen granules and increasing spaces between granules (Fig. 3). Moreover, degradation of starch in acidic gastric fluid may also occur, ${ }^{35,36}$ which could facilitate subsequent digestion of starch in simulated intestinal stage.

There was only a small amount of protein associated with the starch gel. Nevertheless, the simulated gastric digestion increased significantly the in vitro enzymatic digestibility of starch gels in the following simulated intestinal digestion stage. Compared with freshly cooked starch gels, more pinholes were

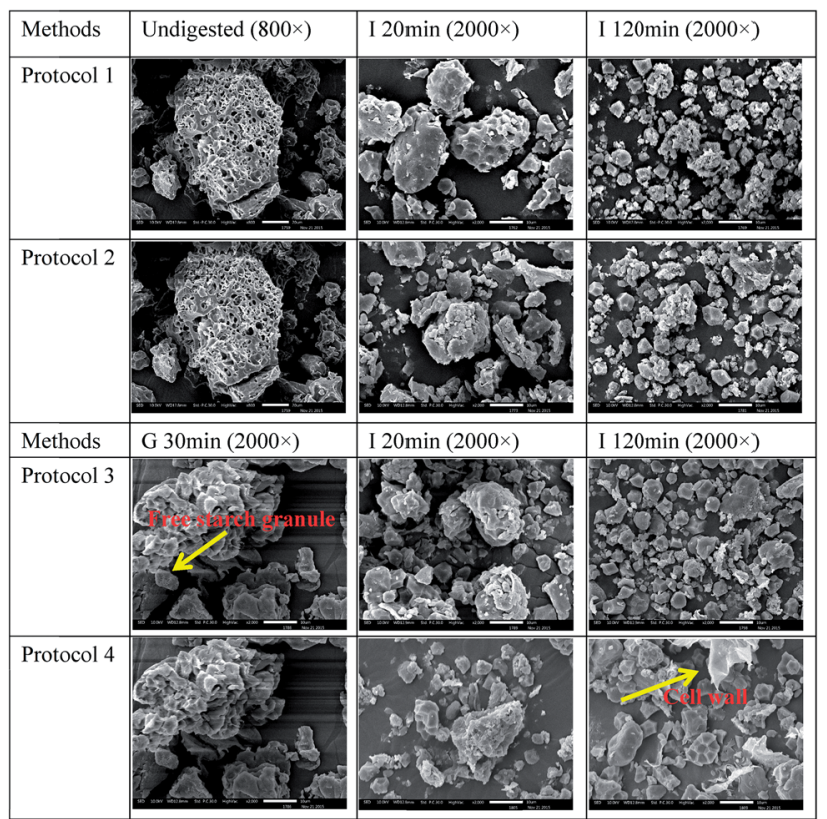

Fig. 3 Scanning electron micrographs of cooked rice before and after digestion. observed on the gel matrix after the pepsin incubation (Fig. 4). These could indicate a loosening of the gel matrix by enzymatic digestion of proteins or partial acid degradation of starch in acidic gastric fluid, both of which could increase the penetration of amylolytic enzymes into starch gels. Removal of proteins associated with the granule surface of native wheat starchenhanced the in vitro digestibility. ${ }^{37}$

\section{Effect of trypsin and chymotrypsin on starch digestibility}

The digestibility of cooked rice and starch gels was increased by the inclusion of trypsin and chymotrypsin in the enzyme mixtures, the effect was most noticeable after the cooked rice substrate has been exposed to the acidic pepsin step (Fig. 2, Table 2). The significant increase in digestibility of starch gels

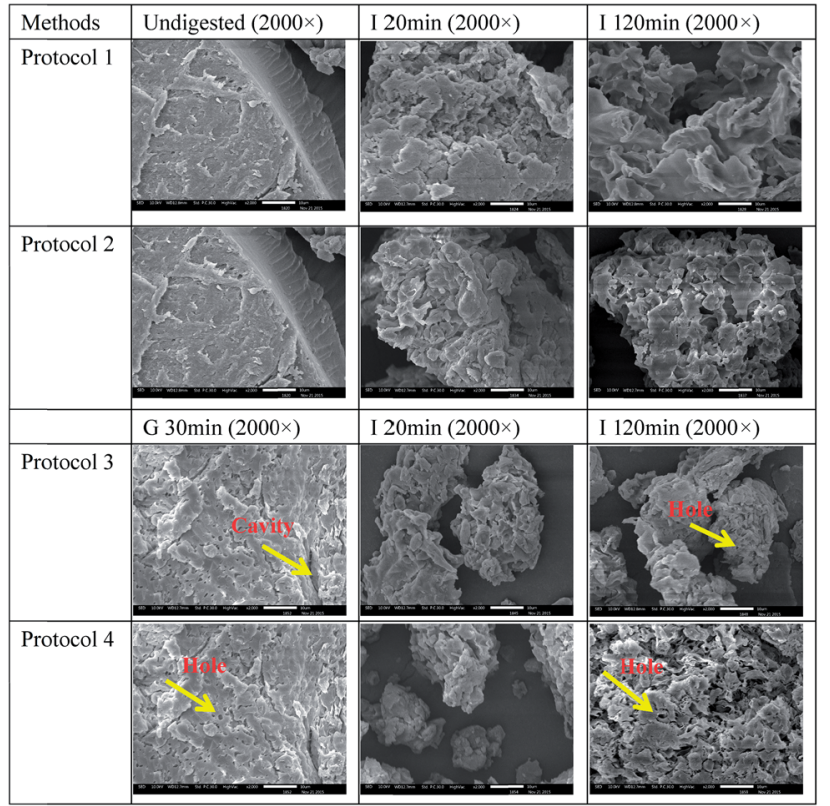

Fig. 4 Scanning electron micrographs of starch gels before and after digestion. 
in the presence of trypsin and chymotrypsin may be attributed to the degradation of granule-associated proteins, which can hinder the access of enzymes to the starch substrate. ${ }^{5}$ The results from the four protocols show that PPA and AMG alone, gave the lowest digestibility of cooked rice and starch gels, while simulated gastric-intestinal digestion with addition of trypsin and chymotrypsin after an incubation with pepsin under acid conditions, gave the highest digestibility.

In this study, the in vitro starch digestibility starch gels was much lower than that of cooked rice, as reflected by the much higher content of RS content in starch gels, the lower $k$ values and $C_{\infty}$ (Table 2). Two possible explanations, not mutually exclusive, are proposed to interpret this observation. Firstly, the protein matrix and cell wall in the rice grains may have resulted in a looser configuration of the swollen starch granules in cooked rice than in the starch gel, allowing easier access for enzymes to the substrate. Another possible explanation may be that starch in cooked rice was gelatinized to a greater extent than starch in gels due to the higher water: starch ratio in rice than in gels, which also increases digestibility. ${ }^{5}$

\section{Kinetics of starch digestibility}

When starch or starch-containing foods are digested in vitro with relatively high enzyme concentrations, the hydrolysis rate decreases with time and plots of the concentration of product formed (or quantity of starch digested) against time are logarithmic. ${ }^{3}$ Therefore, the kinetics can be described by a single rate constant (i.e. first-order kinetics). The kinetic parameters were calculated from the digestograms using a first-order equation. ${ }^{26}$ The concentration of digested starch at the theoretical end of the reaction $\left(C_{\infty}\right)$ and the digestion rate constant $(k)$ of cooked rice and starch gels were obtained from the fit of first-order kinetics for the different protocols as shown in Table 2. The correlation coefficients $\left(R^{2}\right)$ ranging between 0.976 and 0.998 , indicated that all starch digestions follow first-order behavior. The $k$ values of cooked rice followed the order: Protocol $4(0.044)>$ Protocol $3(0.039)>$ Protocol 2 $(0.034) \approx$ Protocol 1 (0.033), the differences between Protocols 1,2 and 3 were not significant. For the starch gels, the $k$ values followed the order of Protocol $4(0.034)>$ Protocol $3(0.015) \approx$ Protocol $2(0.013) \approx$ Protocol 1 (0.010). Compared with cooked rice, starch gels presented much lower $k$ values obtained under the same protocol, indicating that starch gels were digested at a lower rate. A low $k$ value has been proposed to reflect the slow diffusion of amylase into starch samples as digestion proceeds. ${ }^{38}$ The higher $k$ values obtained using Protocol 4 indicated that the digestion rate of cooked rice and starch gels were enhanced greatly by simulating gastric digestion and by the addition of trypsin and chymotrypsin in simulated intestinal digestion.

The values of $C_{\infty}$, which is derived from the first order rate equation and represents the theoretical amount of starch remaining when no more product is formed, differed significantly between cooked rice (95 to 97\%) and starch gels (70 to $83 \%$ ) (Table 2). This indicated that starch gels were less digestible than cooked rice. No significant differences were found in $C_{\infty}$ values between cooked rice, whereas the $C_{\infty}$ values of starch gels obtained using Protocol 3 and 4 were higher than those from Protocol 1 and 2.

\section{Microstructure of cooked rice and starch gels before and after digestion}

The microstructure of cooked rice and starch gels before and after the gastric and gastro-intestinal digestion models are depicted in Fig. 3 and 4. The size of cooked rice samples was reduced gradually during simulated intestinal digestion (Fig. 3, Images I $20 \mathrm{~min}$ and I $120 \mathrm{~min}$ in Protocol 1 and 2). There were considerable changes in morphology of cooked rice samples after simulated gastric digestion (Fig. 3, Image G $30 \mathrm{~min}$ in Protocol 3 and 4), where some free deformed starch granules were observed (yellow arrows). This observation indicated that simulated gastric digestion disrupted the structure of cooked rice. Material from the cell wall (yellow arrow) was clearly observed after simulated gastro-intestinal digestion, indicating that starch and protein matrices were degraded substantially with the occurrence of digestive enzymes-resistant cellulose and hemi-cellulose materials.

Undigested starch gels appeared as a compact material (Fig. 4). After simulated intestinal digestion (Protocol 1 and 2), many cavities or holes were observed on the surface of digesta (Fig. 4), Image I $20 \mathrm{~min}$ and I $120 \mathrm{~min}$. Simulated gastrointestinal digestion resulted in more severe breakdown of the material (Fig. 4, lower images), indicating simulated gastric digestion facilitated digestion of starch gels in the following intestinal digestion, consistent with in vitro digestion results of starch gels (Fig. 2B). Addition of trypsin and chymotrypsin resulted in more severe disruption of starch gels, consistent with the increased digestibility determined by Protocol 4 compared with that determined by Protocol 3. The above results were consistent with starch and protein materials being degraded to a greater extent during simulated gastro-intestinal digestion than during simulated intestinal digestion alone.

\section{Conclusion}

The effect of inclusion of trypsin and chymotrypsin on in vitro enzymatic digestibility of rice starch in simulated intestinal digestion was evaluated. Using only $\alpha$-amylase and amyloglucosidase resulted in the lowest percentage of starch hydrolysis, starch gel hydrolysis was increased to a small extent in a digestion protocol that included an acidic pepsin step. The greatest hydrolysis was obtained when trypsin and chymotrypsin were included in the intestinal digestion following pre-treatment with pepsin. From these results, we conclude that a simulated gastric digestion stage should be performed and, more importantly, trypsin and chymotrypsin included in the simulated intestinal digestion stage. This study provides experimental evidence that using only $\alpha$-amylase and amyloglucosidase in simulated intestinal digestion can lead to the underestimation of the in vitro digestibility of starch. 


\section{Acknowledgements}

The authors gratefully acknowledge the financial support from the National Natural Science Foundation of China (31522043, 31401651) and the financial support by Program for Innovative Research Team in University (IRT IRT_15R49).

\section{References}

1 P. J. Butterworth, F. J. Warren, T. Grassby, H. Patel and P. R. Ellis, Carbohydr. Polym., 2012, 87, 2189-2197.

2 L. Copeland, J. Blazek, H. Salman and M. C. Tang, Food Hydrocolloids, 2009, 23, 1527-1534.

3 I. Goni, A. Garcia-Alonso and F. S. Calixto, Nutr. Res., 1997, 17, 427-437.

4 M. Tamura, J. Singh, L. Kaur and Y. Ogawa, Food Chem., 2016, 191, 98-104.

5 S. Wang and L. Copeland, Food Funct., 2013, 4, 1564-1580.

6 S. J. Hur, B. O. Lim, E. A. Decker and D. J. McClements, Food Chem., 2011, 125, 1-12.

7 M. Tamura, J. Singh, L. Kaur and Y. Ogawa, Food Chem., 2016, 191, 91-97.

8 A. R. Yousefi, S. M. Razavi and A. Norouzy, Food Funct., 2015, 6, 3126-3134.

9 L. T. Coles, P. J. Moughan and A. J. Darragh, Anim. Feed Sci. Technol., 2005, 123-124, 421-444.

10 B. Zhang, K. Wang, J. Hasjim, E. Li, B. M. Flanagan, M. J. Gidley and S. Dhital, J. Agric. Food Chem., 2014, 62, 1482-1491.

11 G. M. Bornhorst and R. Paul Singh, Annu. Rev. Food Sci. Technol., 2014, 5, 111-132.

12 A. Guerra, L. Etienne-Mesmin, V. Livrelli, S. Denis, S. Blanquet-Diot and M. Alric, Trends Biotechnol., 2012, 30, 591-600.

13 M. Minekus, M. Alminger, P. Alvito, S. Ballance, T. Bohn, C. Bourlieu, F. Carriere, R. Boutrou, M. Corredig, D. Dupont, C. Dufour, L. Egger, M. Golding, S. Karakaya, B. Kirkhus, S. Le Feunteun, U. Lesmes, A. Macierzanka, A. Mackie, S. Marze, D. J. Mcclements, O. Menard, I. Recio, C. N. Santos, R. P. Singh, G. E. Vegarud, M. S. Wickham, W. Weitschies and A. Brodkorb, Food Funct., 2014, 5, 1113-1124.

14 B.-H. Lee, L. A. Bello-Pérez, A. H.-M. Lin, C. Y. Kim and B. R. Hamaker, Cereal Chem., 2013, 90, 335-343.

15 P. J. Butterworth, F. J. Warren and P. R. Ellis, Starch, 2011, 63, 395-405.
16 H. N. Englyst, S. M. Kingman and J. H. Cummings, Eur. J. Clin. Nutr., 1992, 46, S33-S50.

17 J. W. Woolnough, J. A. Monro, C. S. Brennan and A. R. Bird, Int. J. Food Sci. Technol., 2008, 43, 2245-2256.

18 Z. A. Syahariza, S. Sar, J. Hasjim, M. J. Tizzotti and R. G. Gilbert, Food Chem., 2013, 136, 742-749.

19 A. Dartois, J. Singh, L. Kaur and H. Singh, Food Biophys., 2010, 5, 149-160.

20 P. A. Sopade and M. J. Gidley, Starch, 2009, 61, 245-255.

21 G. Spigno and D. M. De Faveri, J. Food Eng., 2004, 62, 337344.

22 J. Chrastil, Carbohydr. Res., 1987, 159, 154-158.

23 AACC International Approved Methods of Analysis, 11th Ed. Method 44-15.02 - Air Oven Methods. Approved October 30, 1975. Reapproved November 3, 1999, http://dx.doi.org/ 10.1094/AACCIntMethod44-15.02.

24 S. Lu, T.-T. Cik, C.-y. Lii, P. Lai and H.-H. Chen, LWT-Food Sci. Technol., 2013, 54, 224-228.

25 H.-J. Kang, I.-K. Hwang, K.-S. Kim and H.-C. Choi, J. Agric. Food Chem., 2003, 51, 6598-6603.

26 I. Goni, A. Garcia-Alonso and F. Saura-Calixto, Nutr. Res., 1997, 17, 427-437.

27 M. Frei, P. Siddhuraju and K. Becker, Food Chem., 2003, 83, 395-402.

28 R. J. C. Hsu, S. Lu, Y.-h. Chang and W. Chiang, J. Cereal Sci., 2015, 61, 1-7.

29 M. O. Reed, Y. Ai, J. L. Leutcher and J. L. Jane, J. Food Sci., 2013, 78, H1076-H1081.

30 R. Sagum and J. Arcot, Food Chem., 2000, 70, 109-111.

31 L.-J. Zhu, Q.-Q. Liu, J. D. Wilson, M.-H. Gu and Y.-C. Shi, Carbohydr. Polym., 2011, 86, 1751-1759.

32 J. Singh, A. Dartois and L. Kaur, Trends Food Sci. Technol., 2010, 21, 168-180.

33 W. Zou, M. Sissons, M. J. Gidley, R. G. Gilbert and F. J. Warren, Food Chem., 2015, 188, 559-568.

34 E. H. J. Kim, J. R. Petrie, L. Motoi, M. P. Morgenstern, K. H. Sutton, S. Mishra and L. D. Simmons, Food Biophys, 2008, 3, 229-234.

35 S. Wang, J. Blazek, E. Gilbert and L. Copeland, Carbohydr. Polym., 2012, 87, 1941-1949.

36 S. Wang and L. Copeland, Crit. Rev. Food Sci. Nutr., 2015, 55, 1081-1097.

37 S. Wang, H. Luo, J. Zhang, Y. Zhang, Z. He and S. Wang, J. Agric. Food Chem., 2014, 62, 3636-3643.

38 S. Dhital, A. K. Shrestha and M. J. Gidley, Carbohydr. Polym., 2010, 82, 480-488. 